\title{
ON $n$-WIDTHS OF CERTAIN FUNCTIONAL CLASSES DEFINED BY LINEAR DIFFERENTIAL OPERATORS
}

\author{
Y. MAKOVOZ
}

ABSTRACT. Let $A=D^{r}+\sum_{k=0}^{r-1} a_{k}(t) D^{k}, a_{k} \in C^{k}$, be a differential operator and let $W_{p}(A)$ be the class of functions $x(t)$ for which $\|A x\| \leqslant 1$ in $L_{p}[0,1]$. We prove that the asymptotic behavior of the Kolmogorov widths $d_{n}\left(W_{p}(A), L_{q}\right), 1 \leqslant p, q \leqslant \infty$, when $n \rightarrow \infty$ does not depend on $a_{k}$.

Let $L_{p}^{r}$ be the class of functions $x(t)$ having $x^{(r-1)}$ absolutely continuous on $[0,1]$ and $x^{(r)} \in L_{p}[0,1]$. Suppose

$$
A=D^{r}+\sum_{k=0}^{r-1} a_{k}(t) D^{k}
$$

is a linear differential operator, $a_{k} \in C^{k}[0,1]$ and $W_{p}(A)=\left\{x: x \in L_{p}^{r},\|A x\|_{p} \leqslant 1\right\}$. We shall write $W_{p}(A)=W_{p}^{r}$ if $A=D^{r}$.

The Kolmogorov $n$-width of a set $W$ in a normed linear space $X$ is defined as

$$
d_{n}(W, X)=\inf _{\Gamma_{n}} \sup _{x \in W} \inf _{\gamma \in \Gamma_{n}}\|x-\gamma\|_{X},
$$

where the last infimum is taken over all $n$-dimensional linear subspaces $\Gamma_{n} \subset X$.

The Gelfand $n$-width of $W$ is defined as

$$
d^{n}(W, X)=\inf _{\Gamma^{n}} \sup _{x \in W \cap \Gamma^{n}}\|x\|_{X}
$$

where the infimum is taken over all linear subspaces of codimension $n$.

The widths $d_{n}\left(W_{p}(A), L_{q}\right)$ were studied in several papers [1-4]. In particular, the precise rate of decrease of $d_{n}$ when $n \rightarrow \infty$ was found in [2] for $p=q=\infty$ and in [4] for $p=q=2$ and $p=1, q=2$.

It turned out, in these cases, that the asymptotic behavior of $d_{n}\left(W_{p}(A), L_{q}\right)$ is precisely the same for all operators $A$ of the form (1). The purpose of this paper is to prove that this fact is true for all combinations of $p$ and $q$.

TheOREM. For all $p, q, 1 \leqslant p, q \leqslant \infty$, and $A$

$$
\lim _{n \rightarrow \infty} \frac{d_{n}\left(W_{p}(A), L_{q}\right)}{d_{n}\left(W_{p}^{r}, L_{q}\right)}=1 .
$$

Received by the editors August 25, 1982.

1980 Mathematics Subject Classification. Primary 41A65; Secondary 41A17.

Key words and phrases. n-widths, differential operators. 
A remarkable feature of the simple proof given below is that it requires neither explicit construction of optimal subspaces $\Gamma_{n}$ nor even any specific information on their structure.

Let $L_{p 0}^{r}, L_{p 1}^{r}$ be subsets of $L_{p}^{r}$ consisting of functions $x(t)$ for which $x^{(k)}(0)=0$ or $x^{(k)}(1)=0$ respectively $(k=0,1, \ldots, r-1) ; \tilde{L}_{p}^{r}=L_{p 0}^{r} \cap L_{p 1}^{r} ; \stackrel{0}{W_{p}}(A)=W_{p}(A) \cap$ $L_{p 0}^{r}, \quad \stackrel{1}{W_{p}}(A)=W_{p}(A) \cap L_{p 1}^{r}, \quad \tilde{W}_{p}(A)=W_{p}(A) \cap \tilde{L}_{p}^{r}$. We will write $a_{n} \sim b_{n}$ if $\lim _{n \rightarrow \infty} a_{n} / b_{n}=1 ; C_{1}, C_{2}, \ldots$ will denote positive constants which may depend on $A, r, p, q$ but do not depend on $n$.

We shall need the following lemmas.

LEMMA 1. There exists such $C_{1}, C_{2}$ that for all $x \in L_{p 0}^{r}$,

$$
C_{1}\left\|x^{(r)}\right\|_{p} \leqslant\|A x\|_{p} \leqslant C_{2}\left\|x^{(r)}\right\|_{p} \quad(1 \leqslant p \leqslant \infty) .
$$

The second of these inequalities is obvious; the first follows from the second and the fact that $L_{p 0}^{r}$ is a Banach space in both norms $\left\|x^{(r)}\right\|_{p}$ and $\|A x\|_{p}$.

An immediate consequence of this lemma is that at least the rough order of decrease of $n$-widths for $W_{p}^{r}$ and $W_{p}(A)$ is the same (namely, $n^{-\alpha}, \alpha=\alpha(p, q, r)>0$ ).

The next statement can be obtained by a simple geometric argument.

LEMMA 2. Let

$$
\delta(M, \varepsilon)=\sup \left\{\|x\|_{\infty}:\left\|x^{\prime}\right\|_{\infty} \leqslant M,\|x\|_{1} \leqslant \varepsilon\right\} .
$$

Then $\delta(M, \varepsilon) \rightarrow 0$ if $\varepsilon \rightarrow 0$ for any fixed $M>0$.

LEMMA 3 [5]. There exists such $C_{3}$ that for any $x \in \tilde{L}_{p}^{r}(1 \leqslant p \leqslant \infty), 1 \leqslant k \leqslant r$,

$$
\left\|x^{(k)}\right\|_{p} \leqslant C_{3}\|x\|_{p}^{(r-k) / r} \cdot\left\|x^{(r)}\right\|_{p}^{k / r} .
$$

Before proceeding to the proof of the theorem we note the following important duality:

$$
d_{n}\left(W_{p}(A), L_{q}\right) \sim d^{n}\left(W_{q^{\prime}}\left(A^{\prime}\right), L_{p^{\prime}}\right),
$$

where $p^{\prime}=p \cdot(p-1)^{-1}, q^{\prime}=q \cdot(q-1)^{-1}$ and $A^{\prime}$ is the adjoint differential operator.

To prove (4) it suffices to show that

$$
d_{n}\left(\stackrel{0}{W_{p}}(A), L_{q}\right)=d^{n}\left(\stackrel{1}{W_{q^{\prime}}}\left(A^{\prime}\right), L_{p^{\prime}}\right) .
$$

Indeed, if $x \in W_{p}(A)$ then $x=x_{0}+z$, where $x_{0} \in \mathscr{W}_{p}(A), A z=0$. So $d_{m}\left(\stackrel{0}{W_{p}}(A), L_{q}\right)=d_{m+r}\left(W_{p}(A), L_{q}\right)$. A similar argument can be applied to $W_{q^{\prime}}\left(A^{\prime}\right)$.

Let $\Gamma_{n}$ be an arbitrary $n$-dimensional subspace of $L_{q}$. Then

$$
\sup _{\substack{0 \\ x \in W_{p}(A)}} \inf _{\gamma \in \Gamma_{n}}\|x-\gamma\|=\sup \langle x, y\rangle
$$

where the last supremum is taken over all $x \in \stackrel{0}{W_{p}}(A), y \in L_{p^{\prime}},\|y\|_{p^{\prime}} \leqslant 1, y \perp \Gamma_{n}$. 
We define $u$ by

$$
A^{\prime} u=y, \quad u^{(k)}(1)=0 \quad(k=0,1, \ldots, r-1) .
$$

Then $\langle x, y\rangle=\left\langle x, A^{\prime} u\right\rangle=\langle A x, u\rangle$ and

$$
\sup _{\substack{0 \\ x \in W_{p}(A)}}\langle x, y\rangle=\sup _{\substack{0 \\ x \in W_{p}(A)}}\langle A x, u\rangle=\sup _{\|\zeta\|_{p} \leqslant 1}\langle\zeta, u\rangle=\|u\|_{p^{\prime}} .
$$

It follows from (6) that $u \in \stackrel{1}{W}_{p^{\prime}}\left(A^{\prime}\right)$. The condition $y \perp \Gamma_{n}$ means, in terms of $u$, that $u$ belongs to some subspace of codimension $n$. So, by taking the infimum over $\Gamma_{n}$ in (5) and (7) we have

$$
d_{n}\left(W_{p}(A), L_{q}\right) \geqslant d^{n}\left(W_{q^{\prime}}\left(A^{\prime}\right), L_{p^{\prime}}\right)
$$

The opposite inequality can be obtained by inverting the preceding argument.

It follows from (4) that $d_{n}$ in (2) can be replaced by $d^{n}$. On the other hand, $\tilde{W}_{p}$ is defined by imposing $2 r$ linear conditions on $W_{p}(A)$. This produces at most $2 r$-units shift of the index $n$ in $d^{n}$ which does not affect the asymptotic behavior of $d^{n}$ since its order is $n^{-\alpha}$. So in order to prove the theorem it suffices to prove that

$$
d^{n}\left(\tilde{W}_{p}(A), L_{q}\right) \sim d^{n}\left(\tilde{W}_{p}^{r}, L_{q}\right)
$$

Now, for a subspace $\Gamma^{n}\left(\operatorname{codim} \Gamma^{n}=n\right)$, let

$$
\sup _{x \in \tilde{W}_{p}^{r} \cap \Gamma^{n}}\|x\|_{q}=\varepsilon .
$$

Suppose $x \in \tilde{W}_{p}(A) \cap \Gamma^{n}$. We can assume, without loss of generality, that $\|A x\|_{p}$ $=1$. On the other hand, by Lemma 1 we have $\left\|x^{(r)}\right\|_{p} \leqslant C_{1}^{-1}$, so (9) implies that $\|x\|_{q} \leqslant C_{1}^{-1} \varepsilon_{n}$. Since $x \in \tilde{L}_{p}^{r}$ we also have $\left\|x^{\prime}\right\|_{\infty} \leqslant C_{1}^{-1}$,

$$
\|x\|_{p} \leqslant\|x\|_{\infty} \leqslant \delta\left(C_{1}^{-1}, C_{1}^{-1} \varepsilon\right)=\delta
$$

and, by Lemma 2, $\delta \rightarrow 0$ when $\varepsilon \rightarrow 0$. Combining this fact with Lemma 3 we have $\left\|x^{(k)}\right\|_{p} \rightarrow 0$ when $\varepsilon \rightarrow 0$. So

$$
\eta=\sup _{x \in \tilde{W}_{p}(A)}\left\|A x-x^{(r)}\right\|_{p} \rightarrow 0
$$

if $\varepsilon \rightarrow 0$. Since $x \in(1+\eta) \cdot \tilde{W}_{p}^{r} \cap \Gamma^{n}$ we have $\|x\|_{q} \leqslant \varepsilon(1+\eta)$. So if the subspace $\Gamma^{n}$ is 'good' (in terms of $d^{n}$ ) for $\tilde{W}_{p}^{r}$ it is asymptotically equally good for $\tilde{W}_{p}(A)$.

To prove the opposite statement suppose that

$$
\sup _{x \in \tilde{W}_{p}(A) \cap \Gamma^{n}}\|x\|_{q}=\varepsilon
$$

and let $x \in \tilde{W}_{p}^{r} \cap \Gamma^{n}$. Then, assuming that $\left\|x^{(r)}\right\|_{p}=1$, we have from Lemma 1 that $\|A x\|_{p} \leqslant C_{2}$. So, by a similar argument, $\|x\|_{q} \leqslant C_{2} \varepsilon,\|x\|_{p} \leqslant \delta\left(1, C_{2} \varepsilon\right)$ and the proof can be concluded as above. 


\section{REFERENCES}

1. Charles K. Chui and Phillip W. Smith, Some nonlinear spline approximation problems related to N-widths, J. Approx. Theory 13 (1975), 421-430.

2. C. A. Micchelli and A. Pinkus, On n-widths in $L^{x}$ : Limit as $n \rightarrow \infty$, IBM Research Report RC 5573 , 1975.

3. $\ldots$ On n-widths in $L^{x}$, Trans. Amer. Math. Soc. 234 (1977), 139-174.

4. K. Nasyrova, On asymptotic behavior of certain compacts in the space $L_{2}[0,1]$, Mat. Zametki 20 (3) (1973), 331-339. (Russian)

5. E. M. Stein, Functions of exponential type, Ann. of Math. (2) 65 (1957), 582-592.

Department of Mathematics, OKlahoma State University, Stillwater, Oklahoma 74074 